\title{
Design of Digital Respiration Rate Meter
}

\author{
Ajeya Anand, Akhil Raja K, Greeshma K, Jaseena K, Rahul R \\ Department of Applied Electronics and Instrumentation Engineering \\ Government Engineering College, West Hill, Kozhikode \\ University of Calicut \\ Calicut, India.
}

\begin{abstract}
In this paper, we describe The Digital Respiration Rate Meter, a device used to measure the rate of respiration. It consists of a transmitter section, receiver section and breathing setup. Transmitter section transmits IR waves which are cut by a displacement transducer in the breathing setup. This IR wave is sensed by the receiver section. The Controller section reads this and generates the count to be displayed. This is displayed on three seven-segment displays for read out.
\end{abstract}

\section{General Terms}

Respiration; Rate; Meter.

\section{Keywords}

Digital; Respiration; Rate; Meter.

\section{INTRODUCTION}

Measurement of physical parameters like heart rate and respiration rate is crucial in the field of medicine. Respiration involves inhalation and exhalation. Inhalation is the taking in of air and exhalation is the expulsion of deoxygenated air. These two processes of inhalation and exhalation together constitute ventilation. Respiratory rate (RR) is also known as respiration rate, pulmonary ventilation rate or ventilation rate. This is the count of breaths a living being, such as a human, takes within certain duration of time. This is generally given in breaths per minute.

Recognizing alterations in respiration rate is an early evidence of illness recognition [1]. This may directly or indirectly lead to diagnosis of sickenss. Interstitium are support structure for air sacs in the lungs. It is here in these air sacs that gaseous exchange takes place. Interstitial disease may lead to fast shallow ventilation. This is efficient because the lung with interstitial disease is less compliant, requiring more distending pressure per unit of volume than normal. By breathing at lower tidal volumes, the work of breathing is diminished. Congestive heart failure can produce a similar effect. Any central nervous system depressing drug, or its overdose, will affect the respiratory rate and pattern. Respiration rate is a major vital sign. In healthcare anaesthetized patients are continuously monitored for respiration rate.

\section{EXISTING MEASUREMENT TECHNIQUES}

There exist some techniques for measurement of respiration rate. The human respiration rate is usually measured when a person is at rest and simply involves counting the number of breathes for one minute by counting the number of times the chest rises or falls. Respiration rates may generally increase with illness, or other medical conditions. When checking respiration, it is important to also note whether a person has any difficulty in breathing.

Average respiratory rate reported in a healthy adult at rest is usually given as 12 breaths per minute $(12 / 60 \mathrm{~Hz})$ but estimates do vary between sources [2], e.g., 12-20 breaths per minute, $10-14$, between $16-18$, etc. With such a slow rate, more accurate readings are obtained by counting the number of breaths over a full minute.

The commonly used method for measuring RR is counting the number of respirations per minute. Also triage nurses' measurements [3] of respiratory rate show low sensitivity in detecting Bradypnea (a condition of slow breathing) and Tachypnea (a condition of fast breathing).

Some techniques developed to measure respiration rate include

1. Thermistors placed in front of nostrils

2. Micro-switches or displacement transducers put across the chest

3. Impedance electrodes and

4. Signals from $\mathrm{CO}_{2}$ measurement

But these techniques have some drawbacks such as

1. Low sensitivity

2. Inaccuracy and

3. High cost of implementation and maintenance.

Hence the classic method of counting, by nurse, is preferred, that too only in critical situations.

\section{RELATED WORK}

A design of mobile application for respiratory rate [4] required a physical touch input to help count respiration rate. The requirement of assistant to count the rate could lead to error, also this would not be appropriate for personal use by patient. The digital respiration rate meter simplifies the counting process for use by even illiterate patients.

Use of ultra wideband sensors for respiration rate [5] is better used in emergency rescue systems. The complexity of the method and increased cost in implementation makes it less affordable for large populations which have financial constraints in access to healthcare and diagnosis. 
A comparative study on respiration rate measurement using a counting device and manual measurement shows the benefits of using a digital counting technique [6]. The use of temperature during exhalation and inhalation was used to measure respiration rate in another method [7]. This method requires sensors and communication devices which increase the overall cost of the respiration rate diagnostic instrument. Contact based respiration rate measurements such as impedance based on pneumography [8] are invasive methods and may cause discomfort to the patient under diagnosis.

A method for respiration rate measurement, inspired by low cost design approach by Souvik Das [9] and a construction method published [10] is presented here. The meter can be used to monitor the respiration rate, pulse rate (by using a proper sensor) and heart rate. It responds fast and is costeffective compared to conventional medical equipment. By using this, respiration rate can be measured in the range of 0 999 respirations/minute.

\section{DESCRIPTION}

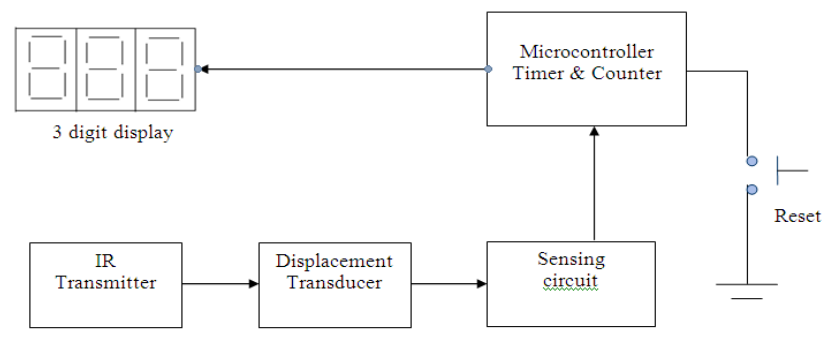

Figure 1. Block diagram

\subsection{BLOCK DIAGRAM DESCRIPTION}

The microcontroller section is implemented using the microcontroller AT89S52 [12]. Power Supply of $+12 \mathrm{~V}$ battery is used as the power to the circuits. IR Receiver is a $38 \mathrm{kHz}$ TSOP 1738 IR Receiver module. Meter reading is read out from three seven-segment LCD displays. An Astable Multivibrator using LM555 IC generates $38 \mathrm{KHz}$. Two BC547 epitaxial silicon transistors are used as Darlington Amplifier. One IR LED transmitter acts as the IR transmitter.

\subsection{CIRCUIT DESCRIPTION}

\subsubsection{Regulator Section}

An LM7805 fixed positive voltage IC is used as regulator. A regulated output of $5 \mathrm{~V}$ is obtained from the regulator section. This is fed to microcontroller.

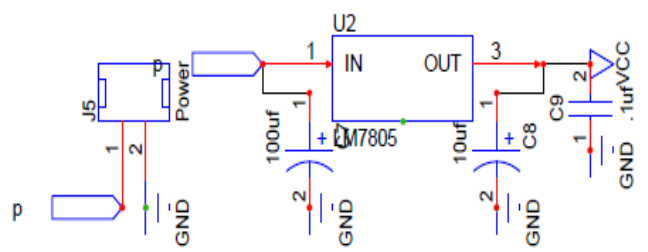

Figure 2. Block diagram

\subsubsection{Microcontroller Section}

The microcontroller (uC) section is the core of the circuit. The microcontroller is programmed to read the count of respiration.

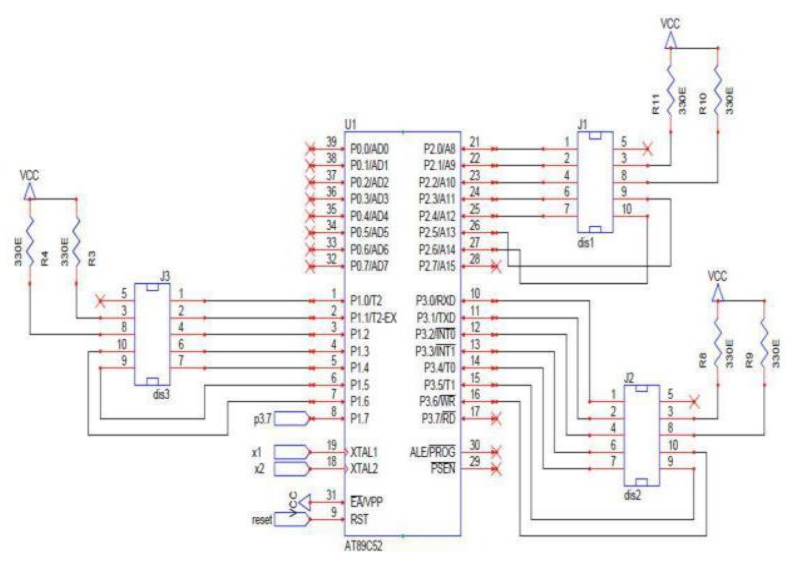

Figure 3. Control section.

The AT89S8252 is a low-power, high-performance CMOS 8-bit microcomputer with $8 \mathrm{~K}$ bytes of downloadable Flash programmable and erasable read only memory and $2 \mathrm{~K}$ bytes of EEPROM The AT89S8252 provides the following standard features: $8 \mathrm{~K}$ bytes of downloadable Flash, $2 \mathrm{~K}$ bytes of EEPROM, 256 bytes of RAM, 32 I/O lines, programmable watch-dog timer, two data pointers, three 16-bit timer/counters, a six-vector two-level interrupt architecture, a full duplex serial port, on-chip oscillator, and clock circuitry.

\subsubsection{IR transmitter Section}

These frequencies are fed to BC547.The collector output of BC547 is modulated using a $38 \mathrm{KHz}$ carrier that produced from LM555. It is then transmitted by using IR Led.

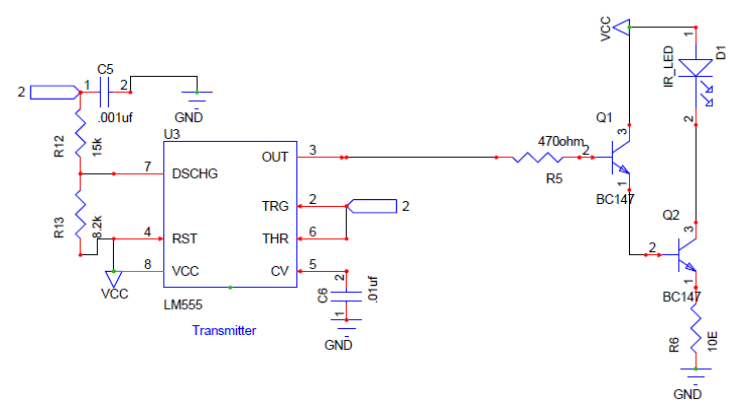

Figure 4. IR Transmitter section.

\subsubsection{IR Receiver Section}

89S52 controller is the main part of receiver. TSOP 1738 is used to receiving incoming signals that produced from the transmitter. The signal is then fed to the microcontroller. Microcontroller then gives the rate to display on seven segment LCD.

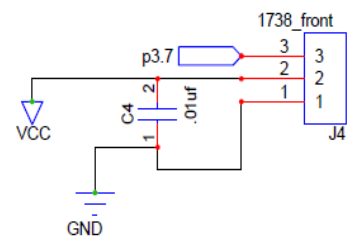

Figure 5. IR Receiver section. 


\subsubsection{Displacement transducer}

Breathing causes up and down displacement of a thermocol ball in a glass tube. This displacement is in correspondence to the rate of respiration. This is the transduction principle. Components used are breathing mask, a glass tube and a thermocol ball.

\section{CONSTRUCTION}

The patient is allowed to breathe through a breathing mask. The glass tube is connected to the valve of the mask. The other end of the tube is restricted so that the ball does not leave the tube, whereas air can easily pass through. The IR LED and receiver are placed in diametrically opposite points on outer surface of glass tube.

\subsection{DESIGN}

Care has been taken so that the entire assembly offers very low resistance to airflow. So with the mask, the patient can breathe as easily as he can without a mask.

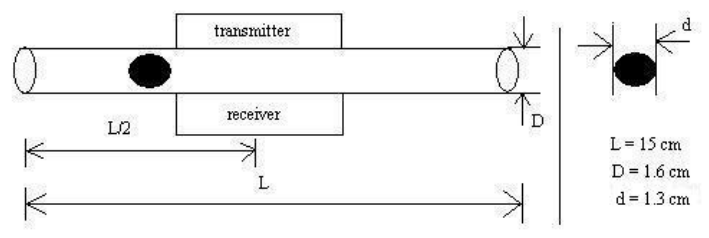

Figure 6. Displacement transducer.

If the glass tube selected, is too short then very shallow respiration, which are negligible, will be counted. If the tube is very long, the device will not be handy. So the device has been designed for an optimum length. A glass tube of diameter $1 \mathrm{~cm}$ and of length $15 \mathrm{~cm}$ is taken. A thermocol ball of diameter $0.7 \mathrm{~cm}$ (approx) is chosen. The IR transmitter and receiver are placed at diametrically opposite points on the surface of the tube, at the middle of the tube.

\section{CONCLUSION}

Here, we have developed a low cost, accurate and effective device to measure respiration rate. The device is compatible with existing life support systems.

The design is fairly easy to implement. It provides good sensitivity and accuracy. The method provides fast response and direct digital output. The digital respiration rate meter is highly portable. The meter is less expensive due to use of IR technique. It has low power consumption and low maintenance cost.

The mechanical part of the digital respiratory rate meter is fragile and must be handled carefully. Also, in case of patients with contagious disease separate mask has to be used. In this case disposable masks could be used to maintain the safety.

The meter can be useful in diagnosing respiratory disorders. It can help in finding relations between ECG, EMG, EOG, EEG etc with respiratory rate. Also, this can help in exploring possibilities in finding out multiple physiological disorders when used in conjunction with other rate measures as mentioned above.

\section{ACKNOWLEDGMENT}

This work was supported by the Faculty of Department of Applied Electronics and Instrumentation Engineering, Government Engineering College Kozhikode, University of Calicut.

\section{REFERENCES}

[1] H Kenneth Walker, MD, W Dallas Hall, MD, and J Willis Hurst, MD, 1990, Clinical Methods, 3rd edition, The History, Physical, and Laboratory Examinations.

[2] 2014, J.S. Peretta, Neonatal and Paediatric respiratory care- A patient case method, F.A.Davis Company.

[3] Consistency of Triage in Victoria's Emergency Departments Guidelines for Triage Education and Practice, July 2001.

[4] D. Dunsmuir , W. Karlen, H. Gan, Michelle Chiu, C.L. Petersen, G.A.Dumont, J.M. Ansermino, The Design of a Respiratory Rate Mobile Application.

[5] Natalia V. Rivera , Swaroop Venkatesh , Chris Anderson, R.Michael Buehrer, Multi-Target Estimation of Heart And Respiration Rates Using Ultra Wideband Sensors.

[6] I.Smith, J. Mackay, N.Fahrid, 2011, Respiratory rate measurement: a comparison of methods, British Journal of Healthcare assistants, Vol 05 No 01.

[7] Archita Agnihotri, 2013 ,"Human Body Respiration Measurement Using Digital Temperature Sensor with I2C Interface", International Journal of Scientific and Research Publications, Volume 3, Issue 3.

[8] A.K. Gupta, 2011, Respiration Rate Measurement Based on Impedance Pneumography, Application Report, Texas Instruments.

[9] Souvik Das, 2013, "Development of a respiration rate meter-a low-cost design approach", Health Informatics An International Journal (HIIJ) Vol.2, No.2.

[10] 2009, Construction: Digital Respiratory Rate Meter, Electronics For You, June, Issue Vol. 41 No. 6

[11] Leslie Cromwell; Fred J Weibell; Erich A Pfeiffer, 2011, Biomedical Instrumentation and Measurements, Prentice Hall.

[12] M. A. Mazidi, J. G. .Mazidi., 2007, The 8051 microcontroller and embedded systems, Pearson.

[13] National Center for Biotechnology Information (NCBI) website www.ncbi.nlm.nih.gov.

[14] The Basic Geriatric Respiratory Examination Medscape www.medscape.com. 